\title{
Commensal Hafnia alvei strain reduces food intake and fat mass in obese mice-a new potential probiotic for appetite and body weight management
}

\author{
Romain Legrand ${ }^{1} \cdot$ Nicolas Lucas $^{1} \cdot$ Manon Dominique ${ }^{1,2,3} \cdot$ Saida Azhar $^{1} \cdot$ Camille Deroissart $^{1,2,3}$. \\ Marie-Anne Le Solliec ${ }^{1,2,3}$. Julie Rondeaux ${ }^{1,2,3}$. Séverine Nobis ${ }^{2,3}$. Charlène Guérin ${ }^{2,3}$ - Fatima Léon ${ }^{3,4}$. \\ Jean-Claude do Rego $0^{3,4}$ - Nicolas Pons ${ }^{5} \cdot$ Emmanuelle Le Chatelier $^{5} \cdot$ S. Dusko Ehrlich $\mathbb{D}^{5} \cdot$ Gregory Lambert $^{1}$. \\ Pierre Déchelotte $\mathbb{C}^{1,2,3,6} \cdot$ Sergueï O. Fetissov ${ }^{1,2,3,7}$
}

Received: 27 April 2019 / Revised: 2 December 2019 / Accepted: 11 December 2019 / Published online: 7 January 2020

(c) The Author(s) 2020. This article is published with open access

\begin{abstract}
Background/objectives Based on the recent identification of E.coli heat shock protein ClpB as a mimetic of the anorexigenic $\alpha$-melanocyte stimulating hormone $(\alpha-\mathrm{MSH})$, the objective of this study was to preclinically validate Hafnia alvei, a ClpBproducing commensal bacterium as a potential probiotic for appetite and body weight management in overweight and obesity.

Methods The involvement of enterobacterial ClpB in the putative anti-obesity effects was studied using ClpB-deficient E.coli. A food-grade H. alvei HA4597 strain synthetizing the ClpB protein with an $\alpha$-MSH-like motif was selected as a candidate probiotic to be tested in $o b / o b$ and high-fat diet (HFD)-fed obese and overweight mice. The relevance of the enterobacterial $\mathrm{ClpB}$ gene to human obesity was studied by in silico analysis of fecal metagenomes of 569 healthy individuals from the "MetaHIT" database.

Results Chronic per os administration of native but not ClpB-deficient E.coli strain reduced body weight gain $(p<0.05)$ and daily meal frequency $(p<0.001)$ in $o b / o b$ mice. Oral gavage of H.alvei for 18 and 46 days in $o b / o b$ and HFD-fed obese mice, respectively, was well tolerated, reduced body weight gain and fat mass in both obesity models $(p<0.05)$ and decreased food intake in hyperphagic $o b / o b$ mice $(p<0.001)$. Elevated fat tissue levels of phosphorylated hormone-sensitive lipase were detected in H.alvei -treated ob/ob mice $(p<0.01)$. Enterobacterial ClpB gene richness was lower in obese vs. non-obese humans ( $\mathrm{p}<0.0001)$ and correlated negatively with BMI in genera of Enterobacter, Klebsiella and Hafnia.

Conclusions H.alvei HA4597 strain reduces food intake, body weight and fat mass gain in hyperphagic and obese mice. These data combined with low enterobacterial $\mathrm{ClpB}$ gene abundance in the microbiota of obese humans provide the rationale for using H.alvei as a probiotic for appetite and body weight management in overweight and obesity.
\end{abstract}

These authors contributed equally: Romain Legrand, Nicolas Lucas

These authors jointly supervised this work: Pierre Déchelotte, Sergueï O. Fetissov

Supplementary information The online version of this article (https:// doi.org/10.1038/s41366-019-0515-9) contains supplementary material, which is available to authorized users.

Gregory Lambert

glambert@targedys.com

$\bowtie$ Sergueï O. Fetissov

Serguei.Fetissov@univ-rouen.fr

Extended author information available on the last page of the article

\section{Introduction}

The etiology of obesity is multifactorial, with the genetic predisposition playing a key role including both the human genome and the metagenomes of multiple microorganisms constituting the body microbiota inhabiting various epithelial surfaces [1]. The gut microbiota is the principal source of bacteria, viruses and fungi in the body with the number of bacteria approximately equal to the total number of human cells [2]. The composition of the gut microbiota changes rapidly due to diet and is influenced by age, sex, geographical location and drugs such as antibiotics [3, 4]. The altered composition of the gut microbiota in obesity has been well documented; its transfer in experimental animals 
was accompanied by the transfer of an obesity phenotype providing proof of its causal role in obesity pathogenesis $[5,6]$. Inversely, a healthy microbiota contribute to the regulation of various physiological processes including the regulation of feeding behavior [7].

It is hence conceivable that the restoration of a healthy microbiota or a modulation of its composition aimed at increasing beneficial bacterial species may represent a strategy for appetite and body weight management in obesity [8]. However, several recent papers reviewed the limited efficiency of traditional probiotic bacteria of the Lactobacillus and Bifidobacterium species in obesity $[9,10]$. A new generation of probiotics should be developed based on the analysis of the gut microbiota composition and a better understanding of the mechanisms of action of commensal bacteria on the host [11]. In this study, we used the recently generated data of specific bacteria-host communication to develop a new potential probiotic for appetite and body weight management in obesity.

The key underlying finding was the identification of E.coli heat shock protein $\mathrm{ClpB}$ as an antigen-mimetic of the anorexigenic $\alpha$-melanocyte stimulating hormone $(\alpha-\mathrm{MSH})$ [12]. Unexpectedly, the same study showed that oral gavage to lean mice with E.coli native but not ClpB-deficient strains decreased their food intake and body weight, suggesting a key role of the ClpB in the anorexigenic effect of E.coli. Considering the key role of $\alpha-\mathrm{MSH}$-mediated melanocortin signaling system in the regulation of energy balance, exemplified by marked hyperphagia and obesity in its deficient states in both rodents and humans, the $\alpha-\mathrm{MSH}$ mimetic properties of bacterial $\mathrm{ClpB}$ suggest that commensal bacteria producing similar with the E.coli $\mathrm{ClpB}$ protein, may be used as an anti-obesity probiotic [13-15].

Thus, the objectives of this study were to demonstrate the relevance of $\mathrm{ClpB}$ protein in potential anti-obesity effects of $\mathrm{ClpB}$ expressing bacteria and to preclinically validate Hafnia alvei (H.alvei) a food-grade, commensal specie of the Hafniaceae (formerly Enterobacteriaceae) family as a putative anti-obesity probiotic [16]. For this purpose, the presence of $\alpha-\mathrm{MSH}$ mimetic epitopes in the ClpB protein of the H.alvei HA4597 strain was analyzed using both in silico and proteomic approaches. Then, we tested H.alvei HA4597 in two mouse models of obesity: genetic, leptindeficient $o b / o b$ mice and nutritional, high-fat diet (HFD)induced obesity. The complementarity of these models is related to the hyperphagia and severe obesity with a standard chow consumption in $o b / o b$ mice combined with moderate obesity in otherwise normo-/hypophagic HFD-fed mice as a model of nutritionally induced overweight. In addition, to test the relevance of $\mathrm{ClpB}$ to the anti-obesity effects, in a separate experiment, ClpB-expressing and ClpB-deficient bacteria were supplied to $o b / o b$ mice. Finally, to further justify the rationale for supplementation of ClpB-expressing probiotic in humans, we performed in silico analysis of the metagenomes from the human fecal microbiota samples of 569 healthy individuals available from the database of the MetaHIT consortium [17] and in which the prevalence of the E.coli $\mathrm{ClpB}$ gene was analyzed in relation to BMI and obesity.

\section{Materials and methods}

\section{Animals}

Animal experiments were approved by the Local Ethical Committee of Normandy (approval N5986). All mice were purchased from Janvier Labs (L'Arbresle, France); they were housed in a specialized animal facility $\left(22 \pm 2{ }^{\circ} \mathrm{C}\right.$, relative humidity $40 \pm 20 \%$ ) under a $12 \mathrm{~h}$ light (7:00 a.m. $-7: 00$ p.m.)/ $12 \mathrm{~h}$ dark cycle. Mice were kept in standard plastic cages $(n=3$ per cage) with ad libitum food (3430 Kliba Nafag standard diet, Kaiseraugst, Switzerland, unless exposed to an HFD) and water access. B6.V-Lep ob/ob JRj mice $(n=75)$ and $\mathrm{C} 57 \mathrm{Bl} / 6 \mathrm{JRj}$ mice $(n=70)$ both males were 6 weeks of age at arrival. After acclimation for 7 days, C57Bl/6 mice had ad libitum access to an HFD with the following caloric content: $45 \%$ fat, $35 \%$ carbohydrates, and $20 \%$ proteins (D12451, Research Diets, New Brunswick, NJ, USA) for 19 weeks. Food intake per cage and individual body weight were measured daily. For the study of the effects of ClpBdeficient E.coli strain on feeding behavior, after 7 days of acclimation $o b / o b$ mice were placed in individual BioDAQ cages (Research Diets).

\section{Experimental procedures in mice}

For the study of the ClpB-deficient E.coli K12 effects, $o b / o b$ mice were randomly divided into three groups $(n=8$ of each) with the same mean body weight. Sample size was estimated based on previous experiments [12]. One group received the E.coli $\mathrm{K} 12$ native strain and another group the E.coli $\mathrm{K} 12 \mathrm{ClpB}$-deficient strain; both strains were received in LB medium and the control group received LB medium only via intragastric gavage as described below for H.alvei The experimental procedure was alike the one described above but mice were kept in individual BioDAQ cages. The E.coli strains and culture conditions have been previously described [12].

For the study of H.alvei HA4597 effects, both $o b / o b$ and C57B16 mice with obesity induced by HFD were randomly divided into two groups $o b / o b(n=12$, in each) and HFD ( $n=22$ in each) with the same mean body weight. Randomization was performed by each cage of three so that the animals from the same cage belonged to the same group. From day 1 onwards, mice received once daily at 6:00 p.m. 
H.alvei HA4597 cultured strain in LB medium or LB medium (control group) in a volume of $10 \mathrm{~mL} / \mathrm{kg}$, via intragastric gavage using a steel drenching cannula (Socorex, Ecublens, Switzerland). Before the gavage, individual body weight and food \& water intakes (by cage) were measured. Abnormal symptoms and behavior (sickness and aggressiveness) were monitored and if they occurred, such animals were removed from the study. Body composition was analyzed using the MiniSpec LF50 (Bruker, Rheinstetten, Germany) before and at the end of the treatment (day 18 for $o b / o b$ and day 46 for HFD mice). Then, mice were terminally anesthetized by intraperitoneal injection of ketamine/xylazine solution $(80 / 10 \mathrm{mg} / \mathrm{kg})$ and a blood sample $(\sim 0.5 \mathrm{~mL})$ was taken via abdominal aorta puncture in aprotinin EDTA $(1 \mathrm{mg} / \mathrm{mL})$ containing tubes (BD Vacutainer, Franklin Lakes, NJ, USA). The tubes were centrifuged at $1500 \times g$ during $20 \mathrm{~min}$, and plasma samples were stored at $-80^{\circ} \mathrm{C}$. The following tissues were dissected and snap-frozen in liquid nitrogen prior to storage at $-80^{\circ} \mathrm{C}$ : hypothalamus, epididymal fat pads, and fecal colon content.

\section{Proteomic analysis of Hafnia alvei HA4597}

The TargEDys proprietary H.alvei HA4597 strain (manufactured by Biodis, Noyant, France) was analyzed for the presence of $\alpha$-MSH-like epitopes using western blot (WB) and immunoprecipitation with $\alpha-\mathrm{MSH}$ antibodies (Delphi Genetic, Charleroi, Belgium). Total protein from H.alvei HA4597 cultures was extracted and processed for WB. Shotgun LC-MS/MS mass spectrometry (Biognosys, Schlieren, Switzerland) was used for the identification of bacterial proteins precipitated by $\alpha$-MSH antibodies after total protein extraction from H.alvei HA4597 and E.coli K12. For the detailed procedure see Supplementary data.

\section{Hafnia alvei HA4597 strain preparation for animal experiments}

The H.alvei HA4597 strain was pre-cultured by incubating under agitation of $100 \mu \mathrm{L}$ of the bacterial stock suspension in $10 \mathrm{~mL}$ of Luria Broth culture medium (LB, Conda, Madrid, Spain) overnight at $37^{\circ} \mathrm{C}$. The optical density of diluted preculture (1:10 vol. in culture medium) was measured using a spectrophotometer at $600 \mathrm{~nm}$ (LB medium served as blank) to adjust the optical density at 0.01 at the beginning of the culture. Then, the bacterial culture was started with LB medium at $37^{\circ} \mathrm{C}$ under agitation. After $4 \mathrm{~h}$ of incubation, H.alvei culture samples were taken and stored at $-20^{\circ} \mathrm{C}$ until use. The number of CFU per animal was determined prior to the gavage consisting of $3 \times 10^{8} \mathrm{CFU} /$ day for $o b / o b$ mice and $4 \times 10^{7} \mathrm{CFU} /$ day for the HFD model.

\section{ClpB protein assay}

Development and validation of an E.coli ClpB immunoassay has been described [18]. In brief, rabbit polyclonal anti-E.coli K12 ClpB antibodies (Delphi Genetics) were coated onto 96-well Maxisorp plates (Nunc, Rochester, NY). One hundred microliters of plasma samples or ClpB protein (Delphi Genetics) standard dilutions were incubated for $2 \mathrm{~h}$ at room temperature (RT). Mouse monoclonal antiE.coli K12 ClpB antibodies (Delphi Genetics) and goat antimouse alkaline phosphatase conjugated IgG (Jackson ImmunoResearch Laboratories Inc., West Grove, USA) were used for ClpB detection and p-nitrophenyl phosphate solution (Sigma-Aldrich, St. Louis, USA) as a substrate. The reaction was stopped by $3 \mathrm{~N} \mathrm{NaOH}$ and optical density was determined at $405 \mathrm{~nm}$ using a microplate reader Metertech 960 (Metertech Inc., Taipei, Taiwan). Concentration was measured by referring to the ClpB protein standard curve.

\section{ClpB DNA in fecal content}

Total DNA was extracted from the colonic fecal content using the QIAamp Mini Spin Columns, following the manufacturer's instructions (Qiagen, Courtaboeuf, France). The PCR conditions and ClpB DNA primers have been described [12], 16 S rRNA gene primers were used from Turner et al. [19].

\section{Phosphorylated hormone-sensitive lipase (pHSL) levels in fat tissue}

To evaluate the possible effects of H.alvei HA4597 supplementation on fat tissue lipolysis, the levels of pHSL protein, a lipolytic marker, were studied by WB in the epididymal fat tissue of both models using anti-pHSL antibodies (Cell Signaling Technology, MA, USA).

\section{Expression of hypothalamic neuropeptides}

To study the possible effects of H.alvei HA4597 supplementation on the hypothalamic feeding-regulatory neuropeptides, mRNA expression of agouti-related protein (AgRP), neuropeptide Y (NPY), and proopiomelanocortin (POMC) were determined by RT-PCR. Total RNA was extracted from mouse hypothalami in TRIZOL reagent according to the supplier instructions (Invitrogen, Carlsbad, CA, USA). RT-PCR was performed using $1 \mu \mathrm{g}$ of total RNA and $200 \mathrm{U}$ of SuperScript II reverse transcriptase (Invitrogen) followed by SYBR Green technology on BioRad CFX96 real-time PCR system (BioRad, Hercules, CA, USA). The PCR primers for the detection of mRNA 
precursors of NPY, POMC, and AgRP have been published [20].

\section{In silico analysis of the presence of the H.alvei and E.coli ClpB gene in human gut microbiota}

In silico screening was performed against the 9,879,896 microbial genes catalog representative of the human intestinal microbiota (IGC catalog) established from metagenomic assembling of 1267 individuals [17], for details see the supplemental data. For this study, only data from 569 healthy individuals (384 European and 185 Chinese) with their annotated BMI were considered. Ethical approvals for the sample collection and informed consent have been obtained by National ethical committee for all studied groups [17]. The amino acid sequence of the chaperone protein $\mathrm{ClpB}$ of E. coli $\mathrm{K} 12$ (also known as heat shock protein F84.1; NCBI accession number: NP_417083.1) was used as query to screen the metagenomic data. The reference catalog was screened at the protein level using Blastp $(v \cdot 2.7 .1+)$ with an $e$-value threshold of $1 \mathrm{E}-5$ and $40 \%$ identity at least over $90 \%$ of protein length. Targeted ClpB encoding gene presence and abundance were measured using IGC abundance table established in $\mathrm{Li}$ et al. [18].

\section{Statistical analysis}

Data were analyzed using GraphPad Prism 5.02 (GraphPad Software Inc., San Diego, CA, USA). Group differences were compared by the analysis of variance (ANOVA) with Tukey's post tests or Kruskal-Wallis test followed by Dunn's post tests according to normality results evaluated by the Kolmogorov-Smirnov test. Individual group differences were analyzed using two-sided Student's $t$-test or Mann-Whitney's test. Dynamics of body weight and food intake changes were analyzed by two-way repeated measurments (RM) ANOVA followed by Bonferroni's post tests. For all tests, $p<0.05$ was considered statistically significant. Results are shown as mean \pm SEM.

\section{Results}

\section{E.coli K12 native but not the ClpB mutant strain decreases body weight gain in obese mice}

A decrease in body weight gain was observed after 4 days of treatment by E.coli $\mathrm{K} 12$ bacteria and persisted throughout the following 16 days of the study (Fig. 1a). Per os administration of mice with the $\mathrm{ClpB}$ mutant E.coli K12 strain had no significant effect on body weight gain (Fig. 1a, b). Total fat mass was also decreased in mice receiving E.coli $\mathrm{K} 12$ native but not the $\mathrm{ClpB}$ mutant strain
(Fig. 1c), the latter was significant when compared directly with the control group (Student's $t$-test $p<0.05$ ). Analysis of feeding behavior revealed that supplementation in E.coli $\mathrm{K} 12$ reduced the total daily food intake as compared with both control and ClpB-deficient strain-fed mice (Fig. 1d). Decrease in total food intake was due to a decrease in the number of meals (Fig. 1e) without changing the meal size (not shown). A weaker anorexigenic effect was observed in mice supplemented with the ClpB mutant strain (Fig. 1d) which was also due to a decrease in meal number (Student's $t$-test $p<0.01$ vs. controls) without affecting meal size (not shown).

\section{Hafnia alvei HA4597 produces ClpB protein with a-MSH-like epitope}

WB on H.alvei extracted proteins using anti- $\alpha-\mathrm{MSH}$ polyclonal antibodies revealed several bands including one at about $96 \mathrm{KDa}$ corresponding to the molecular weight of the full $\mathrm{ClpB}$ molecule, and a further two bands at around 20 and $10 \mathrm{KDa}$ (Fig. 2a).

Using mass spectrometry identification following the immunoprecipitation of H.alvei HA4597 and E.coli K12 total proteins with an anti- $\alpha$-MSH polyclonal antibody, $507 \pm 70$ and $792 \pm 28$ proteins were respectively recovered and 114 and 96 proteins, respectively, were significantly enriched in samples precipitated by $\alpha-\mathrm{MSH}$ antibodies vs. total $\operatorname{IgG}$ (data not shown). ClpB was among the recovered proteins in both strains but it was about 10 times more abundant in H.alvei HA4597 than in E.coli K12 (Fig. 2b). High levels of ClpB recovery by total $\mathrm{IgG}$ was expected due to the natural presence of ClpBreactive $\operatorname{IgG}$ in non-immunized animals and humans [12]. Sequence alignments between $\alpha-\mathrm{MSH}$ and recovered proteins showed that only the ClpB protein from both H.alvei HA4597 and E.coli K12 strains displayed the $\alpha$ MSH-like epitope with the predicted melanocortin-like activity (Fig. 2c). These data were confirmed and further extended by in silico analysis of the bacterial protein reference database (see below).

\section{Hafnia alvei HA4597 reduces body weight gain and fat mass in ob/ob and HFD obese mice}

H.alvei treatment was not accompanied by any adverse effects. In $o b / o b$ mice, body weight gain was significantly lower after 9 days of H.alvei HA4597 gavage resulting in a $50.1 \%$ decrease at the last day of treatment (Fig. 3a). In the HFD mouse model, prior to H.alvei provision, the obesity was induced by HFD (Supplementary Fig. 1). In HFD-fed obese mice, the body weight gain decreased during the H.alvei HA4597 treatment as compared with the control group starting from the 23rd day of treatment (Fig. 3b), with 


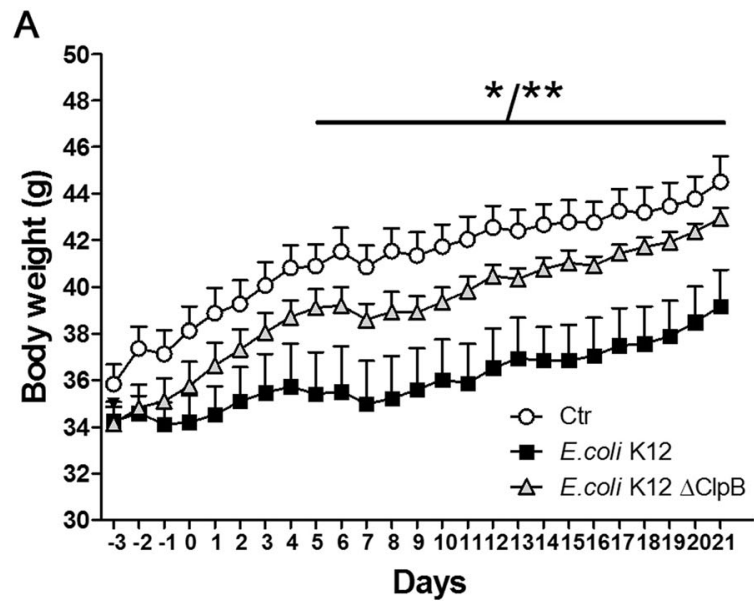

Fig. 1 Effects of E.coli K12 native and ClpB-deficient strains in $o b /$ $\boldsymbol{o b}$ mice. a Body weight dynamics. b Body weight gain (\%). $\mathbf{c}$ Total fat mass. d Daily food intake. e Daily meal number. a 2-Way RM ANOVA $p<0.01$, Bonferroni post tests $* * p<0.01$ for days $6,8,11$,

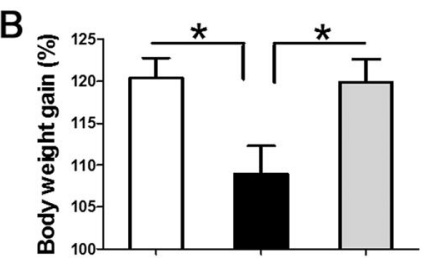

C
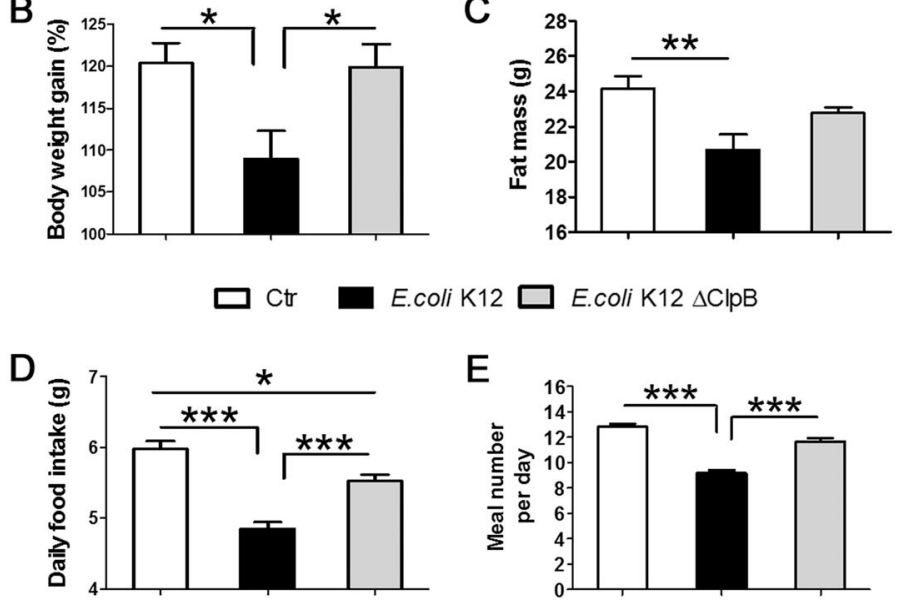

and 12 , other days $* p<0.05$. b ANOVA $p<0.05$, Tukey's post tests $* p<0.05$. c ANOVA $p<0.01$, Tukey's post tests $* * p<0.01$. d ANOVA $p<0.0001$, Tukey's post tests $* * * p<0.001, * p<0.05$.

e Kruskal-Wallis $p<0.0001$, Dunn's post tests $* * * p<0.001$.
A

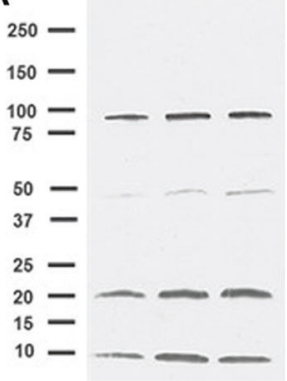

$\begin{array}{lr}\alpha-\mathrm{MSH} & 1- \\ \mathrm{ClpB} \text { H.alvei } & 534- \\ \text { ClpB E.coli } & 534-\end{array}$

B

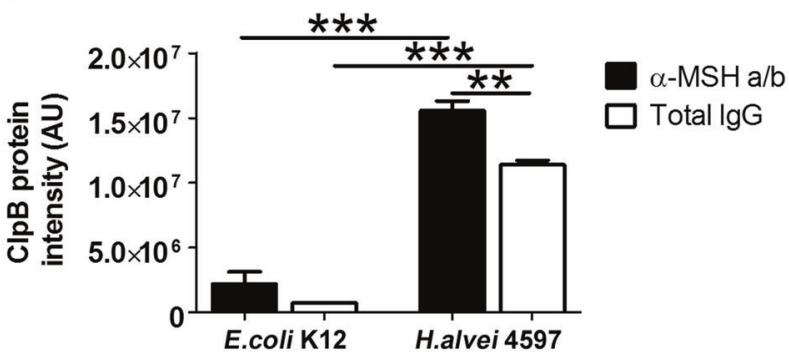

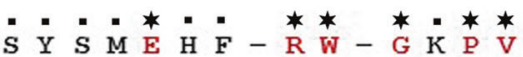

$-13$

V E I A E V L A R W T G I P V S R - 550

A E I A E V L A R W T G P V S R - 550
Fig. 2 Proteomic analysis of $\mathrm{H}$.alvei HA4597 for $\alpha$-MSH-mimetic proteins. a Western blot of H.alvei extracted total proteins using anti$\alpha-\mathrm{MSH}$ antibodies (each lane correspond to a different H.alvei sample). b Intensity of the $\mathrm{ClpB}$ protein recovered from H.alvei and E.coli K12 after immunoprecipitation using rabbit polyclonal $\alpha$-MSH antibodies or total rabbit IgG and identified by mass spectrometry (AU arbitrary units). c Amino acid sequence alignment between the $\alpha$-MSH peptide and the ClpB proteins from H.alvei and E.coli. Stars indicate identical amino acids, periods indicate weak similarity $<0.5$ of Gonnet PAM250 Matrix used in the EMBOS Stretcher program (https://www. ebi.ac.uk/Tools/psa/). b ANOVA, $p<0.0001$, Tukey's post tests $* * * p<0.001, * * p<0.01$. the largest difference observed at day 33 corresponding to a $94.7 \%$ decrease $(0.13 \pm 0.41$ vs. $2.53 \pm 0.43 \mathrm{~g})$. At the last day of treatment this difference decreased to a $38.1 \%$ (2.3 \pm 0.4 vs. $3.8 \pm 0.41 \mathrm{~g}$, Student's $t$-test $p<0.05$ ).

Analysis of the body composition revealed lower gain of fat and loss of lean mass in H.alvei HA4597-treated ob/ob mice (Fig. 3c), corresponding to a $38.3 \%$ and $126.8 \%$ decrease, respectively. A decrease of fat mass gain was also observed in the HFD model, corresponding to $51.9 \%$, while a mean decrease of lean mass (72.4\%) did not reach significance (Fig. 3d).

\section{Hafnia alvei HA4597 reduces food intake in ob/ob mice}

In the hyperphagic ob/ob model, H.alvei HA4597 treatment was accompanied by significantly lower cumulative food intake observed from day 9 and resulting in a $20.8 \%$ decrease in food intake at the last day of the experiment (Fig. 3e). No significant differences of cumulative food intake were observed between the H.alvei HA4597-treated and the control mice in the HFD-induced obesity model 

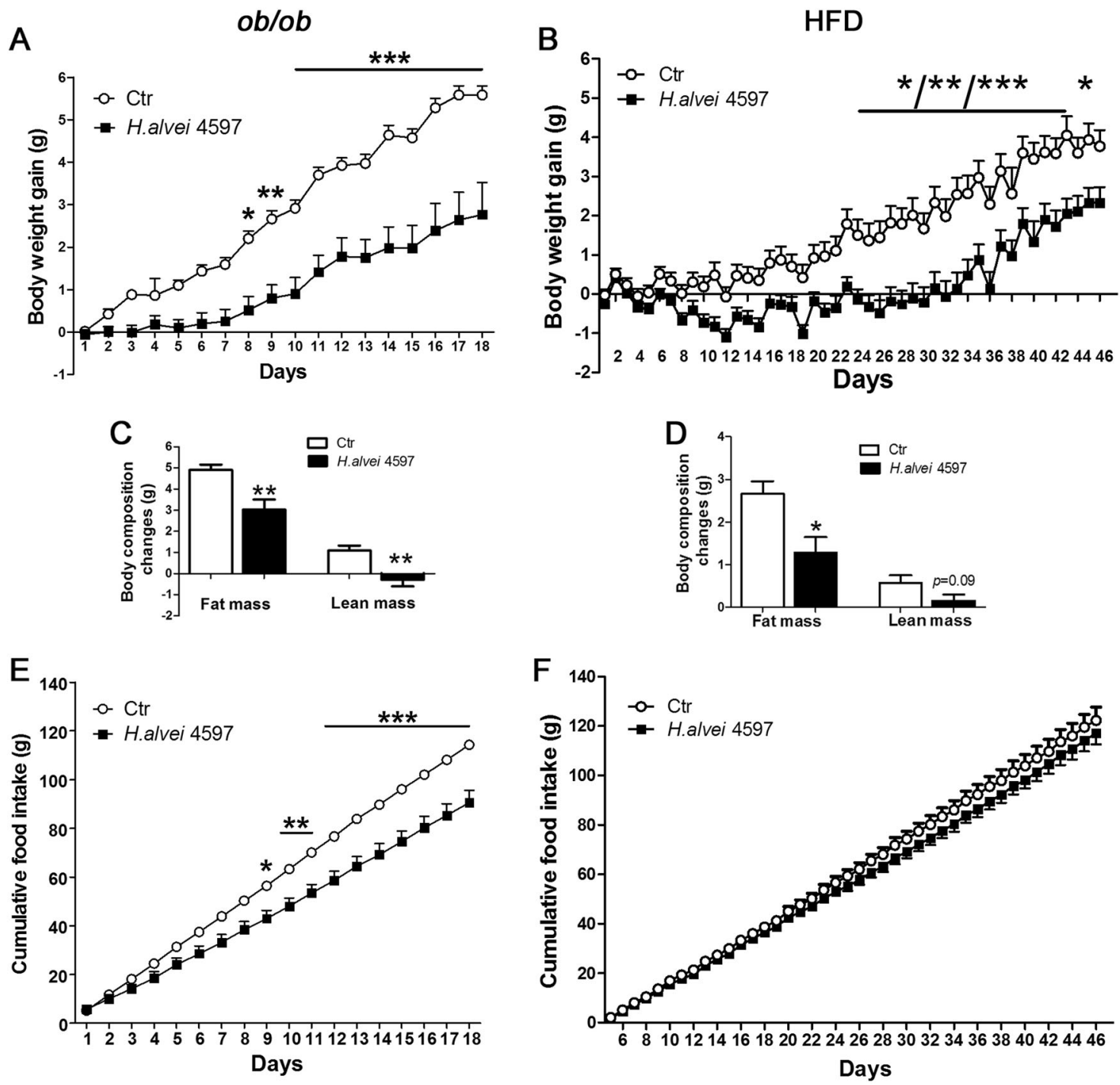

Fig. 3 Effects of H.alvei HA4597 in $o b / o b$ and HFD-fed obese mice on body weight, body composition, and food intake. Body weight dynamics in $o b / o b$ (a) and in HFD-fed obese mice (b). Total fat and lean tissue mass in $o b / o b$ (c) and in HFD-fed obese mice (d). Cumulative food intake in $o b / o b$ (e) and in HFD-fed obese mice (f).

(Kruskal-Wallis test $p=0.29$ ), although a separation of the cumulative food intake curves was visible between the two groups after 4 weeks of treatment (Fig. 3f).

\section{H.alvei HA4597 increases ClpB DNA and plasma levels of ClpB protein in obese mice}

H.alvei provision increased colonic content of ClpB DNA and plasma concentrations of $\mathrm{ClpB}$ protein in $o b / o b$ mice (Fig. 4a). Although a tendency of increased ClpB DNA content was observed in HFD obese mice treated by H.alvei HA4597 (Mann-Whitney test $p=0.09$ ), the plasma concentration of $\mathrm{ClpB}$ protein did not change significantly (Mann-Whitney test $p=0.49$ ), (Fig. 4b).

\section{H. alvei HA4597 increases lipolytic marker in obese mice}

Using the WB, we found that after 18 days of H.alvei treatment in ob/ob mice, the pHSL levels were significantly higher than in the control group (Fig. 4c). In the HFD obese mice treated with H.alvei a trend of increasing pHSL levels was observed (Mann-Whitney test $p=0.08$ ), (Fig. 4d).

\section{H. alvei HA4597 decreases AgRP mRNA in the hypothalamus of obese mice}

We found that in both ob/ob and HFD obese mice, H.alvei HA4597 treatment was accompanied by lower mRNA 

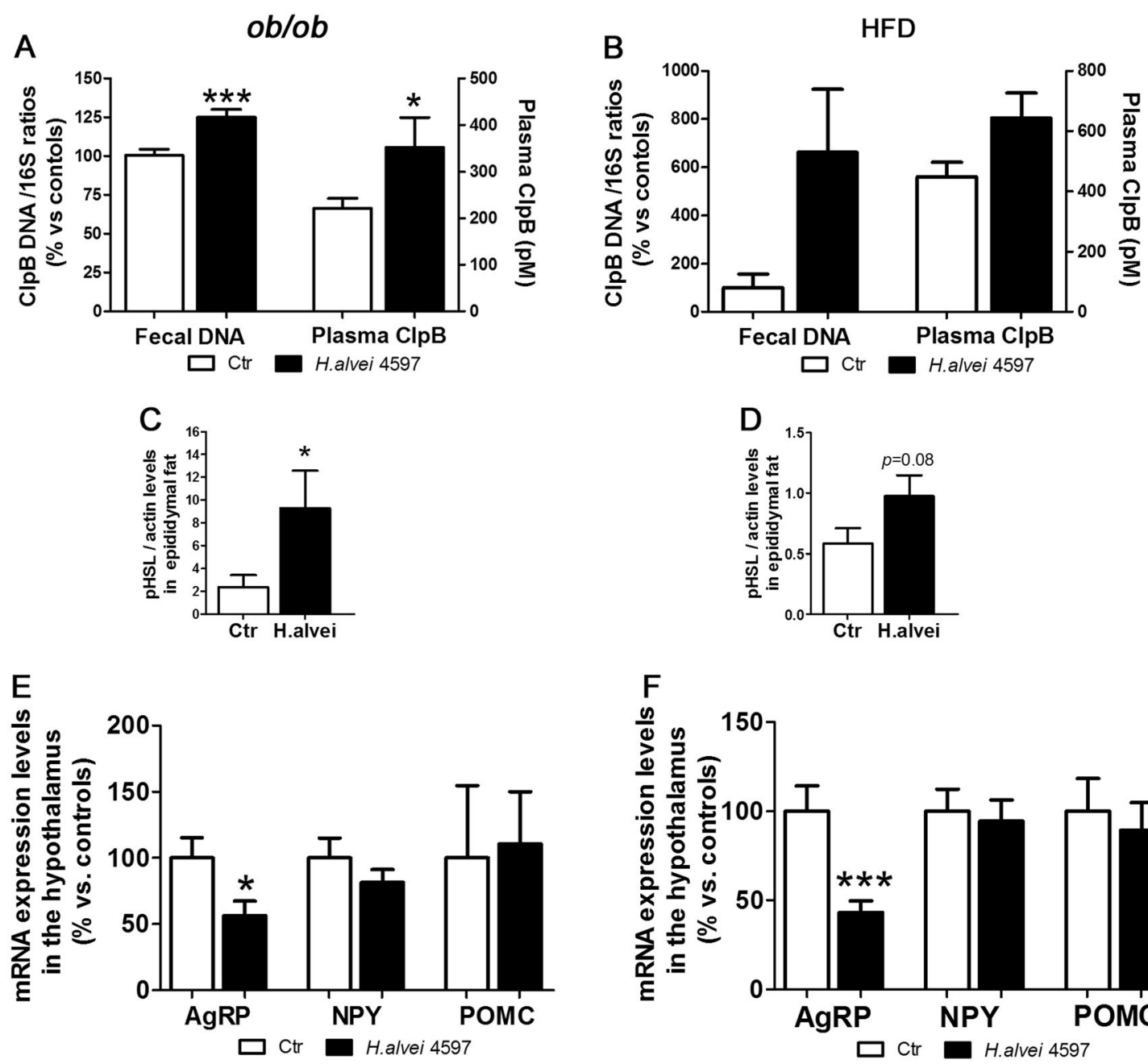

Fig. 4 Effects of H.alvei HA4597 in ob/ob and HFD-fed obese mice on ClpB production, pHSL levels, and hypothalamic neuropeptide mRNA. Levels of ClpB DNA in colonic feces and of ClpB protein in plasma of $o b / o b$ (a) and of HFD-fed obese mice (b). Actin-normalized pHSL levels in the epididymal fat tissue in $o b / o b$ (c) and in HFD-fed

expression levels of an orexigenic neuropeptide AgRP (Fig. 4e, f). However, we did not find significant differences in NPY and POMC mRNA expression levels (Fig. 4e, f).

\section{Enterobacterial ClpB gene is depleted in gut microbiota of obese humans}

Using Blastp, the $10 \mathrm{M}$ protein reference catalog was interrogated for the E.coli $\mathrm{K} 12 \mathrm{ClpB}$ protein sequence. A total of 1527 hits were identified with identity ranging between 40 and $100 \%$. Taxonomic distribution according to sequence identity showed that more than $80 \%$ identity was present in the order Enterobacterales including families of Enterobacteriaceae, Hafniaceae, Morganellaceae and in some unclassified microorganisms (Fig. 5). Moreover, the search for the $\alpha$-MSH-like motif of E.coli ClpB, as shown in Fig. 2c, resulted in its detection in 12 annotated genomic

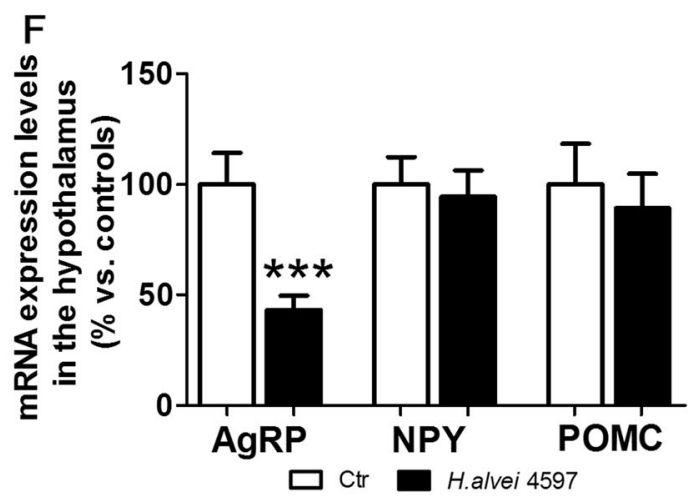

obese mice (d). Hypothalamic mRNA expression levels of AgRP, NPY, and POMC in $o b / o b$ (c) and in HFD-fed obese mice (d) relative to controls $(100 \%)$. a Fecal DNA, Student's $t$-test $* * * p<0.001$. a, e, f Mann-Whitney tests, ${ }^{*} p<0.05, * * * p<0.001$.

records all corresponding to the Enterobacteriales order and represented by species of Escherichia coli, Citrobacter portucalensis, Enterobacter cloacae, Enterobacter xiangfangensis, Klebsiella pneumoniae, Klebsiella michiganensis, Klebsiella oxytoca, Klebsiella aerogenes, Proteus mirabilis, and Hafnia paralvei (Supplementary Table 1). Of note, there is no specie annotated directly to Hafnia alvei in the catalog.

When the Enterobacterales ClpB gene species richness were analyzed for their relation to BMI, the microbiota of obese individuals was found to be depleted from these genes as compared with lean and overweight individuals $(p<0.0001)$ but there were no significant differences between the latter groups (Fig. 6a). However, the main contribution of such difference was due to high richness of the Enterobacterales $\mathrm{ClpB}$ genes in the Chinese cohort as compared with Europeans (Fig. 6b). Nevertheless, it is of 


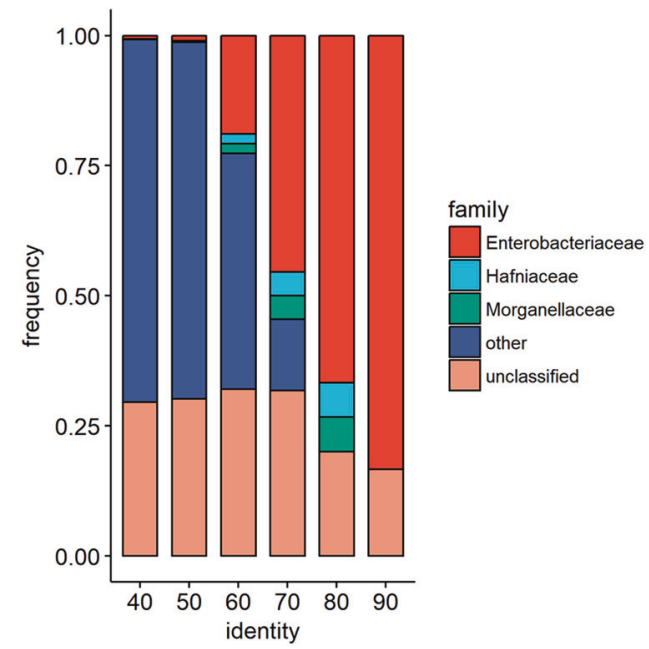

Fig. 5 Taxonomic distribution at family level of E.coli ClpB homology according to sequence identity (from 40 to $90 \%$ ). Note that all identified families with more that $80 \%$ homology belong to the order of Enterobacteriales. Genus Hafnia belongs to the new family of Hafniaceae and genus Proteus to the new family of Morganellaceae.

interest that mean BMI values in both lean and overweight groups were significantly lower in Chinese vs. European cohorts $(21.29 \pm 0.15$ vs. $22.39 \pm 0.14$, Mann-Whitney test, $\quad p<0.0001$ and $26.49 \pm 0.1 \quad$ vs. $27.32 \pm 0.2$, Mann-Whitney test, $p<0.01$, respectively). Within the European cohort, only a tendency of increased ClpB gene richness in lean vs. obese group was observed (Fig. 6c). No significant differences were found between three BMI groups within the Chinese cohort, although the obese group was underrepresented having only two individuals (Fig. 6b). To avoid potential bias, Chinese cohort has been excluded in the BMI correlation analysis. Significant negative correlations within the European cohort were found between BMI and abundance of four Enterobacterales ClpB species. These included Enterobacter xiangfangensis, Klebsiella michiganensis 1, Klebsiella michiganensis 2, and Hafnia paralvei (Spearman's rho $-0.10, p=0.04$ and rho $-0.11, p=0.02$, rho $-0.12, p=$ 0.02 , and rho $-0.12, p=0.02$, respectively, Supplementary Fig. 2). These four species were significantly enriched in lean individuals compared with obese individuals (FDR adjusted $p=0.04$ ).

Although Hafnia alvei specie was not detected in the IGC reference catalog which lacks its full genomic annotation, the closed phylogenetically Hafnia paralvei (msp_1240) was weakly represented. Only 13 healthy individuals harbored this specie in their gut microbiota. Using an alternative bioinformatics approach, blasting of the H.alvei HA4597 specie genome resulted in its detection in metagenome of eight healthy humans i.e., it represents a relatively rare commensal gut specie.
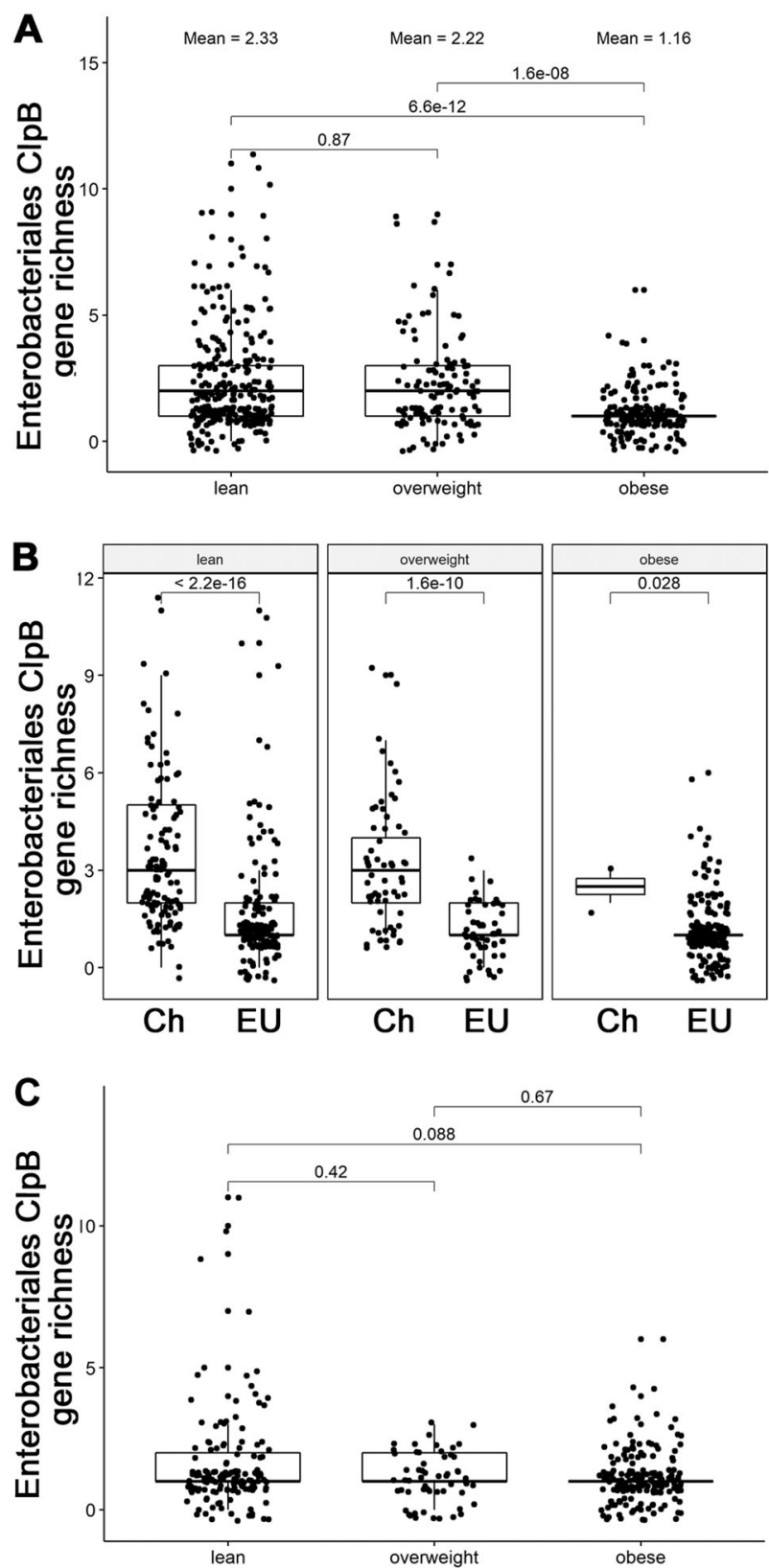

Fig. 6 Enterobacteriales ClpB gene richness in human gut microbiota according to BMI status. a Total combined cohort. b Chinese $(\mathrm{Ch})$ and European (EU) cohorts. c European cohort. $P$ values for the Mann-Whitney test are shown.

\section{Discussion}

Bacteria of the genus Hafnia were identified and named in 1954 by Danish microbiologist Møller after the Latin name for Copenhagen [21]. The food origin of H.alvei was documented by its isolation from raw milk and subsequently from cheese and is considered as part of normal milk microbiota [22, 23]. High numbers of H.alvei have been reported in Camembert cheese, up to $10^{8}$ of viable bacteria per $g$ [24]. Since the nineties, H.alvei has been 
added to milk during the manufacturing of soft cheese due to its beneficial cheese ripening properties. As a result, these bacteria have been consumed naturally and in high amounts for decades by the general population. There have been no adverse effects identified in immunocompetent individuals associated with cheese-derived H.alvei intake, therefore, justifying it as a safe food-grade bacteria annotated by The International Dairy Federation for Microbial Food Cultures [25]. Furthermore, its random presence in the gut microbiota of healthy humans justifies the appellation of H.alvei as a gut commensal.

It is of relevance for the present study to note that H.alvei is the only commensal specie from the Enterobacteriales order to date to carry a food-grade status. In fact, although some other species such as E.coli or Enterobacter are common members of the human gut microbiota, they do not have food-grade status and their presence in food products is considered as a marker of fecal microbial contamination [26]. Therefore, the aforementioned food-grade properties of H.alvei as well as the expression of the $\mathrm{ClpB}$ gene with the same $\alpha$-MSH-like motif as in E.coli served for its selection and validation as a potential probiotic strain for appetite and body weight management in the present study.

Anti-obesity probiotic should be able to interfere with at least two major pro-obesity factors such as hyperphagia and fat tissue accumulation [27]. In this aspect, activation of specific melanocortin receptors (MCR) by $\alpha$-MSH represents a well-established pharmacological target triggering both anorexigenic and lipolytic effects [28]. Indeed, a recent report showed a marked anti-obesity effect of an $\alpha-\mathrm{MSH}$ synthetic peptide analog setmelanotide in two patients with congenital obesity caused by POMC deficiency [29]. Thus, as long as safe and efficient pharmacological anti-obesity compounds continue being developed, a probiotic with natural $\alpha$-MSH mimetic properties may become an option for obesity and overweight prevention or treatment.

In the present study, we confirmed that the $\alpha$-MSH-like motif, initially described in E.coli $\mathrm{K} 12 \mathrm{ClpB}$ is conserved in the Enterobacteriales order including the Hafnia genus and that the H.alvei HA4597 strain synthetizes the $\alpha$ MSH-mimetic ClpB protein. The recovery of some other bacterial proteins by immunoprecipitation with $\alpha-\mathrm{MSH}$ antibodies was due to their binding $\alpha-\mathrm{MSH}$-non related or possibly shorter $\alpha$-MSH-like motifs which did not contain the melanocortin core sequence i.e., they cannot a priori activate the MCR. For instance, a mimicry of the $\alpha-\mathrm{MSH}$ $\mathrm{N}$-terminal was previously identified in the elongation factor-G of E.coli by the Roth's group using corticotropin antibodies [30]. We were also able to detect this protein in $H$.alvei after precipitation with an $\alpha-\mathrm{MSH}$ antibody but it was about 600 times less abundant than the ClpB (data not shown). We also showed for the first time that per os administration of a laboratory E.coli strain from the same with H.alvei Enterobacteriales order in ob/ob mice decreases their food intake, fat mass and body weight gain and that $\mathrm{ClpB}$ was the main active substance responsible for these effects. Although the exact mechanisms of action of $\mathrm{ClpB}$ on the regulation of appetite and energy metabolism remain to be established, the $\alpha$-MSH-mimetic properties may play a key role because the appetitereducing effects of traditional probiotics Lactobacillus and Bifidobacterium, known to express ClpB-like proteins with no homology to $\alpha$-MSH [31], have not been reported in either animal or human studies [9]. With regard to the possible ClpB action sites, it activated the hypothalamic anorexigenic POMC neurons [18] and a recent report showed the capacity of $\mathrm{ClpB}$ to stimulate secretion of peptide YY, a satiety hormone from the gut $[32,33]$. The underlying receptors and intracellular pathways still need to be determined, but the ability of the $\alpha$-MSH-like motif of ClpB to activate cAMP release by type one MCR has been shown [34] pointing to the spatial complementarity between this ClpB peptide fragment and the MCRs.

Our data revealed that H.alvei HA4597 displays the desired probiotic properties of an anti-obesity or weight loss supplement i.e., produce anorexigenic and lipolytic effects in hyperphagic $o b / o b$ mice resulting in decreased fat mass and body weight gain. The body weight and fat tissue lowering effects were also observed in HFD obese mice, although without significant anorexigenic and lipolytic actions. Considering low grade obesity and hypophagia in the HFD obesity model, moderate anorexigenic and lipolytic actions of H.alvei HA4597 may cumulate to reduce total adiposity. In fact, decreased levels of AgRP mRNA expression in the hypothalamus were found in both $o b / o b$ and HFD mice indicating inhibition of the brain orexigenic circuitry by H.alvei. AgRP neurons are known to be inhibited by PYY [35] and, hence, may transmit intestinal satietogenic effects triggered by H.alvei ClpB. Indeed, intestinal epithelium including the enteroendocrine cells was shown to express MCR [36].

Finally, the low abundance of $\mathrm{ClpB}$ gene expressing Enterobacterales species found in the microbiota of obese subjects in the present in silico analysis may indicate insufficient anorexigenic signaling from the gut microbiota to the host, further providing the rationale for supplementation of commensal bacteria expressing the $\mathrm{ClpB}$ protein with an $\alpha-\mathrm{MSH}$-like motif. These results are in agreement with previous reports showing a low prevalence of Enterobacteriaceae in obese individuals [37]. Thus, our study has validated H.alvei HA4597 food-grade bacterial strain as a new potential anti-obesity and anti-overweight probiotic because the desired effects of lowering appetite and/or body weight have been obtained in two mouse models of obesity and overweight. 
Acknowledgements The authors would like to thank Antonia Trower and Clémentine Picolo, Managers at TargEDys SA., for their help in manuscript writing. SF is supported by TMP of Inserm.

\section{Compliance with ethical standards}

Conflict of interest NL, RL, SA, CD, and MALS were full-time employees of TargEDys SA. SOF and PD are co-founders and consultants of TargEDys SA. GL is the CEO of TargEDys SA. Other coauthors declare no conflict of interest.

Publisher's note Springer Nature remains neutral with regard to jurisdictional claims in published maps and institutional affiliations.

Open Access This article is licensed under a Creative Commons Attribution 4.0 International License, which permits use, sharing, adaptation, distribution and reproduction in any medium or format, as long as you give appropriate credit to the original author(s) and the source, provide a link to the Creative Commons license, and indicate if changes were made. The images or other third party material in this article are included in the article's Creative Commons license, unless indicated otherwise in a credit line to the material. If material is not included in the article's Creative Commons license and your intended use is not permitted by statutory regulation or exceeds the permitted use, you will need to obtain permission directly from the copyright holder. To view a copy of this license, visit http://creativecommons. org/licenses/by/4.0/

\section{References}

1. Rosenbaum M, Knight R, Leibel RL. The gut microbiota in human energy homeostasis and obesity. Trends Endocrinol Metab. 2015;26:493-501.

2. Sender R, Fuchs S, Milo R. Revised estimates for the number of human and bacteria cells in the body. PLoS Biol. 2016;14: e1002533.

3. Yatsunenko T, Rey FE, Manary MJ, Trehan I, Dominguez-Bello MG, Contreras M, et al. Human gut microbiome viewed across age and geography. Nature. 2012;486:222-7.

4. Bolnick DI, Snowberg LK, Hirsch PE, Lauber CL, Org E, Parks $\mathrm{B}$, et al. Individual diet has sex-dependent effects on vertebrate gut microbiota. Nat Commun. 2014;5:4500.

5. Cotillard A, Kennedy SP, Kong LC, Prifti E, Pons N, Le Chatelier E, et al. Dietary intervention impact on gut microbial gene richness. Nature. 2013;500:585-8.

6. Turnbaugh PJ, Ley RE, Mahowald MA, Magrini V, Mardis ER, Gordon JI. An obesity-associated gut microbiome with increased capacity for energy harvest. Nature. 2006;444:1027-131.

7. Fetissov SO. Role of the gut microbiota in host appetite control: bacterial growth to animal feeding behaviour. Nat Rev Endocrinol. 2017;13:11-25.

8. Muscogiuri G, Cantone E, Cassarano S, Tuccinardi D, Barrea L, Savastano S, et al. Gut microbiota: a new path to treat obesity. Int J Obes Suppl. 2019. https://doi.org/10.1038/s41367-019-0011-7.

9. Kobyliak N, Conte C, Cammarota G, Haley AP, Styriak I, Gaspar $\mathrm{L}$, et al. Probiotics in prevention and treatment of obesity: a critical view. Nutr Metab (Lond). 2016;13:14.

10. Crovesy L, Ostrowski M, Ferreira DMTP, Rosado EL, SoaresMota M. Effect of Lactobacillus on body weight and body fat in overweight subjects: a systematic review of randomized controlled clinical trials. Int J Obes. 2017;41:1607.

11. Cani PD, de Vos WM. Next-generation beneficial microbes: the case of akkermansia muciniphila. Front Microbiol. 2017;8:1765.
12. Tennoune $\mathrm{N}$, Chan $\mathrm{P}$, Breton J, Legrand $\mathrm{R}$, Chabane $\mathrm{YN}$, Akkermann $\mathrm{K}$, et al. Bacterial $\mathrm{ClpB}$ heat-shock protein, an antigen-mimetic of the anorexigenic peptide [alpha]-MSH, at the origin of eating disorders. Transl Psychiatry. 2014;4:e458.

13. Yaswen L, Diehl N, Brennan MB, Hochgeschwender U. Obesity in the mouse model of pro-opiomelanocortin deficiency responds to peripheral melanocortin. Nat Med. 1999;5:1066-70.

14. Krude H, Biebermann H, Luck W, Horn R, Brabant G, Gruters A. Severe early-onset obesity, adrenal insufficiency and red hair pigmentation caused by POMC mutations in humans. Nat Genet. 1998;19:155-7.

15. Farooqi IS, Keogh JM, Yeo GSH, Lank EJ, Cheetham T, O'Rahilly S. Clinical spectrum of obesity and mutations in the melanocortin 4 receptor gene. N Engl J Med. 2003;348:1085-95.

16. Adeolu M, Alnajar S, Naushad S, S. Gupta R. Genome-based phylogeny and taxonomy of the 'Enterobacteriales': proposal for Enterobacterales ord. nov. divided into the families Enterobacteriaceae, Erwiniaceae fam. nov., Pectobacteriaceae fam. nov., Yersiniaceae fam. nov., Hafniaceae fam. nov., Morganellaceae fam. nov., and Budviciaceae fam. nov. Int J Syst Evol Microbiol. 2016;66:5575-99.

17. Li J, Jia H, Cai X, Zhong H, Feng Q, Sunagawa S, et al. An integrated catalog of reference genes in the human gut microbiome. Nat Biotechnol. 2014;32:834.

18. Breton J, Tennoune N, Lucas N, François M, Legrand R, Jacquemot $\mathrm{J}$, et al. Gut commensal E.coli proteins activate host satiety pathways following nutrient-induced bacterial growth. Cell Metab. 2016;23:1-11.

19. Turner S, Pryer KM, Miao VPW, Palmer JD. Investigating deep phylogenetic relationships among cyanobacteria and plastids by small subunit rRNA sequence analysis1. J Eukaryotic Microbiol. 1999;46:327-38

20. Hamze Sinno M, Do Rego JC, Coëffier M, Bole-Feysot C, Ducrotte P, Gilbert D, et al. Regulation of feeding and anxiety by $\alpha-\mathrm{MSH}$ reactive autoantibodies. Psychoneuroendocrinology. 2009;34:140-9.

21. Moller V. Distribution of amino acid decarboxylases in Enterobacteriaceae. Acta Pathol Microbiol Scand. 1954;35:259-77.

22. Gaya P, Medina M, Nuntez M. Enterobacteriaceae, coliforms, faecal coliforms and salmonellas in raw ewes'milk. J Appl Bacteriol. 1987;62:321-6.

23. Tornadijo E, Fresno JM, Carballo J, Martín-Sarmiento R. Study of Enterobacteriaceae throughout the manufacturing and ripening of hard goats' cheese. J Appl Bacteriol. 1993;75:240-6.

24. Richard J, Zadi H. Inventaire de la flore bactérienne dominante des Camemberts fabriqués avec lait cru. Le Lait, INRA Ed. 1983;63:25-42.

25. Federation ID. Safety demonstration of microbial food cultures (MFC) in fermented food products (Annex 4). Bull Int Dairy Fed. 2012;455:68.

26. Mogren L, Windstam S, Boqvist S, Vågsholm I, Söderqvist K, Rosberg AK, et al. The hurdle approach-a holistic concept for controlling food safety risks associated with pathogenic bacterial contamination of leafy green vegetables. A Review. Front Microbiol. 2018;9:1965.

27. Richard D. Cognitive and autonomic determinants of energy homeostasis in obesity. Nat Rev Endocrinol. 2015;11:489.

28. Anderson EJP, Çakir I, Carrington SJ, Cone RD, GhamariLangroudi M, Gillyard T, et al. 60 YEARS OF POMC: regulation of feeding and energy homeostasis by $\alpha-\mathrm{MSH}$. J Mol Endocrinol. 2016;56:T157-T74.

29. Kühnen P, Clément K, Wiegand S, Blankenstein O, Gottesdiener K, Martini LL, et al. Proopiomelanocortin deficiency treated with a melanocortin-4 receptor agonist. $N$ Engl J Med. 2016;375:240-6. 
30. Qiang X, Liotta AS, Shiloach J, Gutierrez JC, Wang H, Ochani M, et al. New melanocortin-like peptide of E. coli can suppress inflammation via the mammalian melanocortin-1 receptor (MC1R): possible endocrine-like function for microbes of the gut. NPJ Biofilms Microb. 2017;3:31.

31. Fetissov SO, Legrand R, Lucas N. Bacterial protein mimetic of peptide hormone as a new class of protein-based drugs. Curr Med Chem. 2019;26:546-53.

32. Dominique M, Breton J, Guérin C, Bole-Feysot C, Lambert G, Déchelotte $\mathrm{P}$, et al. Effects of macronutrients on the in vitro production of $\mathrm{ClpB}$, a bacterial mimetic protein of $\alpha-\mathrm{MSH}$ and its possible role in the satiety signaling. Nutrients. 2019;11:2115.

33. Cox HM, Tough IR, Woolston A-M, Zhang L, Nguyen AD, Sainsbury A, et al. Peptide YY is critical for acylethanolamine receptor Gpr119-induced activation of gastrointestinal mucosal responses. Cell Metab. 2010;11:532-42.
34. Ericson MD, Schnell SM, Freeman KT, Haskell-Luevano C. A fragment of the Escherichia coli $\mathrm{ClpB}$ heat-shock protein is a micromolar melanocortin 1 receptor agonist. Bioorg Med Chem Lett. 2015;25:5306-8.

35. Batterham RL, Cowley MA, Small CJ, Herzog H, Cohen MA, Dakin CL, et al. Gut hormone PYY(3-36) physiologically inhibits food intake. Nature. 2002;418:650-4.

36. Panaro Brandon L, Tough Iain R, Engelstoft Maja S, Matthews Robert T, Digby Gregory J, Møller Cathrine L, et al. The melanocortin-4 receptor is expressed in enteroendocrine $\mathrm{L}$ cells and regulates the release of peptide $\mathrm{YY}$ and glucagon-like peptide 1 in vivo. Cell Metab. 2014;20:1018-29.

37. Million M, Angelakis E, Maraninchi M, Henry M, Giorgi R, Valero R, et al. Correlation between body mass index and gut concentrations of Lactobacillus reuteri, Bifidobacterium animalis, Methanobrevibacter smithii and Escherichia coli. Int $\mathrm{J}$ Obes. 2013;37:1460-6.

\section{Affiliations}

\section{Romain Legrand ${ }^{1} \cdot$ Nicolas Lucas $^{1} \cdot$ Manon Dominique ${ }^{1,2,3} \cdot$ Saida Azhar $^{1}$ - Camille Deroissart ${ }^{1,2,3}$. Marie-Anne Le Solliec ${ }^{1,2,3}$ - Julie Rondeaux ${ }^{1,2,3}$ - Séverine Nobis ${ }^{2,3}$. Charlène Guérin ${ }^{2,3}$ - Fatima Léon ${ }^{3,4}$. Jean-Claude do Rego $o^{3,4}$. Nicolas Pons ${ }^{5}$. Emmanuelle Le Chatelier ${ }^{5}$. S. Dusko Ehrlich $\mathbb{1}^{5} \cdot$ Gregory Lambert $^{1}$. Pierre Déchelotte $\mathbb{I}^{1,2,3,6} \cdot$ Sergueï O. Fetissov ${ }^{1,2,3,7}$}

1 TargEDys SA, University of Rouen, 76183 Rouen, France

2 Inserm UMR1073, Nutrition, Gut and Brain Laboratory, 76183 Rouen, France

3 Institute for Research and Innovation in Biomedicine (IRIB), University of Rouen Normandy, 76183 Rouen, France

4 Animal Behavioral Platform SCAC, University of Rouen Normandy, 76183 Rouen, France
5 MGP MetaGénoPolis, INRA, Université Paris-Saclay, 78350 Jouy en Josas, France

6 Rouen University Hospital, CHU Charles-Nicolle, 76183 Rouen, France

7 Laboratory of Neuronal and Neuroendocrine Differentiation and Communication, Inserm UMR1239, Mont-Saint-Aignan, France 